(c) Elsevier/INRA

Original article

\title{
Inequality of maximum a posteriori estimators with equivalent sire and animal models for threshold traits
}

\author{
M Mayer \\ University of Nairobi, Department of Animal Production, \\ PO Box 29053, Nairobi, Kenya
}

(Received 25 May 1994; accepted 17 May 1995)

\begin{abstract}
Summary - It is demonstrated that the use of equivalent sire and animal models to describe the underlying variable of a threshold trait leads to different maximum a posteriori estimators. In the simple example data sets examined in this study, the use of an animal model shrank the maximum a posteriori estimators towards zero compared with the estimators under a sire model. The differences between the 2 estimators were particularly noticeable when the heritability on the underlying scale was high and they increased with increasing heritability. Moreover, it was shown that even the ranking of breeding animals may be different when applying the 2 different estimators as ranking criteria.
\end{abstract}

threshold trait / genetic evaluation / maximum a posteriori estimation

Résumé - Inégalité entre des estimateurs du maximum a posteriori avec des modèles équivalents père et animal pour des caractères à seuil. Il est démontré que l'utilisation des modèles équivalents père et animal, pour décrire la variable sous-jacente d'un caractère $\grave{a}$ seuil, conduit à différents estimateurs du maximum a posteriori. Dans les exemples simples analysés dans cette étude, en comparant l'utilisation de ces 2 modèles on constate que les estimateurs du maximum a posteriori tendent $\grave{a}$ se rapprocher de 0 lorsqu'on utilise le modèle animal. Les différences entre les 2 estimateurs sont particulièrement notables quand l'héritabilité sur l'échelle sous-jacente est élevée, et elles augmentent avec l'héritabilité. De plus, il est aussi montré que même le classement des reproducteurs peut être différent selon l'estimateur utilisé comme critère de classement.

caractère à seuil / évaluation génétique / estimation du maximum a posteriori

\section{INTRODUCTION}

New procedures based on the threshold concept have recently been developed for the genetic analysis of ordered categorical data in animal breeding. The 
methods introduced by Gianola and Foulley (1983), Stiratelli et al (1984) and Harville and Mee (1984) are essentially the same and were derived mainly in a Bayesian framework. With these methods, location parameters in the underlying scale are estimated by maximizing a joint posterior density. The estimators are therefore designated as maximum a posteriori estimators. Studies on the properties of maximum a posteriori estimators and comparisons with the estimators and predictors of linear model methods have previously been based on sire models to describe the variable on the underlying scale (eg, Meijering and Gianola, 1985; Höschele, 1988; Weller et al, 1988; Renand et al, 1990; Mayer, 1991). For continuously distributed traits the use of an animal model is the state of the art for the genetic evaluation of breeding animals. A logical step would be to use an animal model as well to describe the underlying variable in genetic evaluation with threshold traits. In the present paper some properties of maximum a posteriori estimators are studied in the context of applying an animal model to describe the underlying variable of threshold traits.

\section{METHODS}

\section{Example data set}

Consider the following simple data structure (2 sires, each with 1 progeny) for a dichotomous character with the 2 realizations $M_{0}(0)$ and $M_{1}(1)$ :
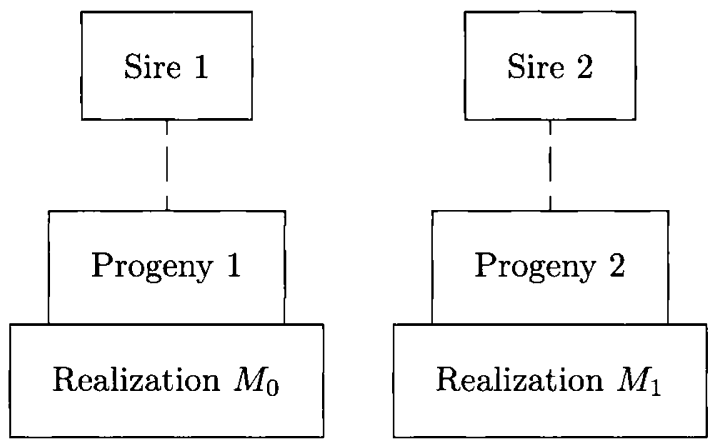

Progeny 1 exhibits the realization $M_{0}$ and progeny 2 the realization $M_{1}$. Assume that we want to estimate the breeding values of the sires for the trait $M$.

\section{Maximum a posteriori estimation}

To estimate the breeding values of the sires, the threshold concept is used and we follow the Bayesian approach along the lines introduced by Gianola and Foulley (1983), Stiratelli et al (1984), and Harville and Mee (1984). Let the data be arranged as a $2 \times 2$ contingency table, where the 2 rows represent, as usual, the sire effects, and the 2 columns represent the realizations $M_{0}$ and $M_{1}$. The elements of the contingency table in the $i$ th row and $j$ th column are designated as $n_{i j}$. The columns indicate mutually exclusive and exhaustive ordered categories of response. 
The $r$ th experimental unit in row $i$ is characterized by an underlying continuously distributed variable, which is rendered discrete through a fixed threshold.

Let us assume that the variance components in the description of the underlying variable are known, at least to proportionality. Using a 'flat' prior for the threshold $(t)$, so as to mimic the traditional mixed model analysis, and assuming that the sire effects on the underlying scale $\left(u_{1}, u_{2}\right)$ a priori are normally, identically and independently distributed with mean 0 and variance $\sigma_{u}^{2}$, the joint posterior density has the form $\left(c_{1}=\right.$ constant $)$ :

$$
g\left(t, u_{1}, u_{2} \mid n_{i j}, \sigma_{u}^{2}, \sigma_{e}^{2}\right)=c_{1} p_{1}\left(1-p_{2}\right) \exp \left\{-\left(u_{1}^{2}+u_{2}^{2}\right) / 2 \sigma_{u}^{2}\right\}=g(\beta)
$$

Using a probit-transformation and a purely additive genetic model for the underlying variable ( $\Phi$ : standardized normal cumulative density function):

$$
p_{i}=\Phi\left(t+u_{i}\right)=\Phi\left(\Theta_{i}\right) \quad i=1,2
$$

The residual standard deviation $\left(\sigma_{e}\right)$ is taken as the unit of measurement on the underlying scale, so that $\sigma_{e}=1$.

The maximum a posteriori estimators are the values which maximize the joint posterior density $g(\beta)$. This is equivalent to the maximation of the log-posterior density, which can be done more easily. The first derivatives of the log-posterior density with respect to $t$ and $u_{i}$ are not linear functions of these parameters and must be solved iteratively using numerical techniques such as Newton-Raphson or Fisher's scoring procedure. The Newton-Raphson algorithm leads to the following iterative scheme:

$$
\left[\begin{array}{ccc}
q_{1}+q_{2} & q_{1} & q_{2} \\
q_{1} & q_{1}+\alpha & 0 \\
q_{2} & 0 & q_{2}+\alpha
\end{array}\right]^{(k-1)}\left[\begin{array}{c}
t \\
u_{1} \\
u_{2}
\end{array}\right]^{(k)}=\left[\begin{array}{l}
s_{1}+s_{2}+q_{1} t+q_{2} t+q_{1} u_{1}+q_{2} u_{2} \\
s_{1}+q_{1} t+q_{1} u_{1} \\
s_{2}+q_{2} t+q_{2} u_{2}
\end{array}\right]^{(k-1)}
$$

where ( $\phi$ : standardized normal probability density function):

$$
\begin{aligned}
s_{1} & =\phi\left(\Theta_{1}\right) / \Phi\left(\Theta_{1}\right) \\
s_{2} & =-\phi\left(\Theta_{2}\right) /\left(1-\Phi\left(\Theta_{2}\right)\right) \\
q_{i} & =s_{i}\left(s_{i}+\Theta_{i}\right) \\
\alpha & =1 / \sigma_{u}^{2}
\end{aligned}
$$

The joint posterior density $g(\beta)$ is symmetric in the sense that $g\left(t=x_{1}\right.$, $\left.u_{1}=x_{2}, u_{2}=x_{3} \mid n_{i j}, \sigma_{u}^{2}, \sigma_{e}^{2}\right)=g\left(t=-x_{1}, u_{1}=-x_{3}, u_{2}=-x_{2} \mid n_{i j}, \sigma_{u}^{2}, \sigma_{e}^{2}\right)$. From this expression it follows directly that the posterior density has its maximum where $t=0$ and $u_{1}+u_{2}=0$. Making use of this result, the iterative equation [2] reduces to:

$$
u_{i}^{(k)}=\left[\left(s_{i}+q_{i} u_{i}\right) /\left(q_{i}+\alpha\right)\right]^{(k-1)}
$$

When using the Fisher-scoring algorithm $q_{i}$ in the above iterative scheme, equation [3] has to be replaced by:

$$
q_{i}=\phi^{2}\left(\Theta_{i}\right) /\left[\Phi\left(\Theta_{i}\right)\left(1-\Phi\left(\Theta_{i}\right)\right)\right]
$$


The estimated breeding values of the sires equal $2 u_{i}^{(k)}$ at convergence. For compatibility reasons with the maximum a posteriori estimators in the following section, which are based on an animal model to describe the underlying variable and where the environmental standard deviation $\left(\sigma_{\varepsilon}\right)$ is taken as the unit of measurement, the estimator in [3] may also be expressed in units of $\sigma_{\varepsilon}$ by multiplying $u_{i}^{(k)}$ by $[(1-4 c) /(1-c)]^{-1 / 2}$ or $\left(1-3 \sigma_{u}^{2}\right)^{-1 / 2}$, respectively, where $c$ is the intraclass correlation $\sigma_{u}^{2} /\left(1+\sigma_{u}^{2}\right)$. Moreover, it is convenient to express the maximum a posteriori estimators in terms of the phenotypic standard deviation $\sigma_{p}=\left(\sigma_{u}^{2}+\sigma_{e}^{2}\right)^{1 / 2}$ by multiplying the estimator from $[3]$ by $[1 /(1-c)]^{-1 / 2}$ or $\left(\sigma_{u}^{2}+1\right)^{-1 / 2}$, respectively.

\section{Use of an equivalent animal model}

Up to now we have dealt with a sire model to describe the underlying variable for the threshold trait. Let the rows of the contingency table now represent the progenies in which the observations are made and we use an equivalent animal model to describe the basis variable and to estimate the breeding values of the sires. For that purpose let $\nu^{\prime}=\left[\nu_{1} \nu_{2} \nu_{3} \nu_{4}\right]$, where $\nu_{1}$ and $\nu_{2}$ represent the additive genetic values on the underlying scale of the progenies 1 and 2 , and $\nu_{3}$ and $\nu_{4}$ represent the additive genetic effects of their sires 1 and 2 .

Under these conditions $\nu$ is a priori normally distributed with mean 0 and variance-covariance matrix $\mathbf{A} \boldsymbol{\sigma}_{a}^{2}$, where $\mathbf{A}$ is the numerator relationship matrix and $\boldsymbol{\sigma}_{a}^{2}$ the additive genetic variance on the underlying scale. For the example data set the relationship matrix has the following form:

$$
\mathbf{A}=\left[\begin{array}{cccc}
1 & 0 & \frac{1}{2} & 0 \\
0 & 1 & 0 & \frac{1}{2} \\
\frac{1}{2} & 0 & 1 & 0 \\
0 & \frac{1}{2} & 0 & 1
\end{array}\right]
$$

The likelihood function invariably follows a product binomial distribution. So the joint posterior density is now:

$$
g\left(t, \nu \mid n_{i j}, \boldsymbol{\sigma}_{a}^{2}, \boldsymbol{\sigma}_{\varepsilon}^{2}\right)=c_{2} p_{1}\left(1-p_{2}\right) \exp \left\{-2\left(\nu_{1}^{2}+\nu_{2}^{2}+\nu_{3}^{2}+\nu_{4}^{2}-\nu_{1} \nu_{3}-\nu_{2} \nu_{4}\right) / 3 \sigma_{a}^{2}\right\}
$$

with

$$
p_{i}=\Phi\left(t+\nu_{i}\right)=\Phi\left(\Theta_{i}\right) \quad i=1,2
$$

and the environmental variance $\left(\sigma_{\varepsilon}\right)$ as the unit of measurement on the underlying scale.

For the joint posterior density [5] the Newton-Raphson algorithm leads after a little algebra to the following iterative equation system: 


$$
\begin{aligned}
{\left[\begin{array}{ccccc}
q_{1}+q_{2} & q_{1} & q_{2} & 0 & 0 \\
q_{1} & q_{1}+\alpha & 0 & 0 & 0 \\
q_{2} & 0 & q_{2}+\alpha & 0 & 0 \\
0 & -1 / 2 & 0 & 1 & 0 \\
0 & 0 & -1 / 2 & 0 & 1
\end{array}\right]^{(k-1)} } & {\left[\begin{array}{c}
t \\
\nu_{1} \\
\nu_{2} \\
\nu_{3} \\
\nu_{4}
\end{array}\right]^{(k)} } \\
& =\left[\begin{array}{l}
s_{1}+s_{2}+q_{1} t+q_{2} t+q_{1} \nu_{1}+q_{2} \nu_{2} \\
s_{1}+q_{1} t+q_{1} \nu_{1} \\
s_{2}+q_{2} t+q_{2} \nu_{2} \\
0 \\
0
\end{array}\right]^{(k-1)}
\end{aligned}
$$

where:

$$
\begin{aligned}
s_{1} & =\phi\left(\Theta_{1}\right) / \Phi\left(\Theta_{1}\right) \\
s_{2} & =-\phi\left(\Theta_{2}\right) /\left(1-\Phi\left(\Theta_{2}\right)\right) \\
q_{i} & =s_{i}\left(s_{i}+\Theta_{i}\right) \\
\alpha & =1 / \sigma_{a}^{2}
\end{aligned}
$$

It can be easily seen that for $\nu_{1}^{(k)}$ and $\nu_{2}^{(k)}$ respectively, this equation system is formally very similar to the iterative equation [2]. Further, it shows the result that $\nu_{3}^{(k)}=\nu_{1}^{(k)} / 2$ and $\nu_{4}^{(k)}=\nu_{2}^{(k)} / 2$, ie the estimated breeding values of the sires equal half of the estimated genetic effects of their progenies. To express the maximum a posteriori estimators in units of the phenotypic standard deviation they have to be multiplied, as in the case of the sire model by $[1 /(1-c)]^{-1 / 2}$ or $\left(\boldsymbol{\sigma}_{a}^{2}+1\right)^{-1 / 2}$, respectively.

\section{More complex data structure}

A more complex data structure, viz the hypothetical data set already used by Gianola and Foulley (1983) to illustrate the maximum a posteriori estimation procedure, was employed to demonstrate the differences between the estimators based on either a sire or an animal model. The data consist of calving ease scores from 28 male and female calves born in 2 herd-years from heifers and cows mated to 4 sires. Calving ease was scored as a response in 1 of 3 ordered categories (normal birth, slight difficulty, extreme difficulty). The data are arranged into a contingency table in table I.

Two different heritability values on the underlying scale $\left(h^{2}=0.20\right.$ and $\left.h^{2}=0.60\right)$ were postulated. For the maximum a posteriori estimation of the location parameters of the underlying variable, the computer program package TMMCAT (Mayer, 1991, 1994a) was used.

\section{Different sire rankings}

Because of the inequality of estimated breeding values stemming from equivalent models to describe the underlying variable, there arose the question as to whether 
Table I. Distribution of calving ease scores by herd-year, age of dam, sex of calf and sire of calf (hypothetical data set by Gianola and Foulley, 1983).

\begin{tabular}{llllllr}
\hline \multirow{2}{*}{ Herd-year } & Age of dam & Sex of calf & Sire of calf & \multicolumn{3}{c}{ Category of response $^{\mathrm{a}}$} \\
& & & & 1 & 2 & 3 \\
\hline 1 & Heifer & Male & 1 & 1 & 0 & 0 \\
1 & Heifer & Female & 1 & 1 & 0 & 0 \\
1 & Cow & Male & 1 & 1 & 0 & 0 \\
1 & Heifer & Female & 2 & 0 & 1 & 0 \\
1 & Cow & Male & 2 & 1 & 0 & 1 \\
1 & Cow & Female & 2 & 3 & 0 & 0 \\
1 & Heifer & Male & 3 & 1 & 1 & 0 \\
1 & Cow & Female & 3 & 0 & 1 & 0 \\
1 & Cow & Male & 3 & 1 & 0 & 0 \\
2 & Heifer & Female & 1 & 2 & 0 & 0 \\
2 & Heifer & Male & 1 & 1 & 0 & 0 \\
2 & Cow & Male & 1 & 0 & 0 & 1 \\
2 & Heifer & Female & 2 & 1 & 0 & 1 \\
2 & Cow & Male & 2 & 1 & 0 & 0 \\
2 & Heifer & Female & 3 & 0 & 1 & 0 \\
2 & Cow & Male & 3 & 0 & 0 & 1 \\
2 & Heifer & Male & 4 & 0 & 1 & 0 \\
2 & Heifer & Female & 4 & 1 & 0 & 0 \\
2 & Cow & Female & 4 & 2 & 0 & 0 \\
2 & Cow & Male & 4 & 2 & 0 & 0 \\
\hline
\end{tabular}

${ }^{\mathrm{a}}$ 1: normal birth; 2 : slight difficulty; 3 : extreme difficulty.

even the ranking of the sires may be different depending upon the model type chosen. To examine this question, data for 3 sires were systematically generated and analyzed. The number of observations $n_{i j}$ of sire $i(i=1,2,3)$ in response category $j(j=1,2)$ was varied from 0 to 8 . A simple random one-way model (sire model, animal model) for the underlying variable and a heritability value of 0.60 was postulated. Again for the analyses, the computer program TMMCAT was used.

\section{RESULTS}

\section{Example data set}

Figure 1 shows the relationship between the estimated breeding value (EBV) of sire 1 and heritability for each of the models. The upper graph represents the estimates taking the environmental standard deviation as the unit of measurement, while in the lower graph the estimates are expressed in units of the phenotypic standard deviation.

It is obvious that the EBV is different whether a sire model or an equivalent animal model is used to describe the underlying variable. The difference between the EBVs is noticeable at a heritability of about 0.60 and increases with increasing heritability. With the phenotypic standard deviation as the unit of measurement, 


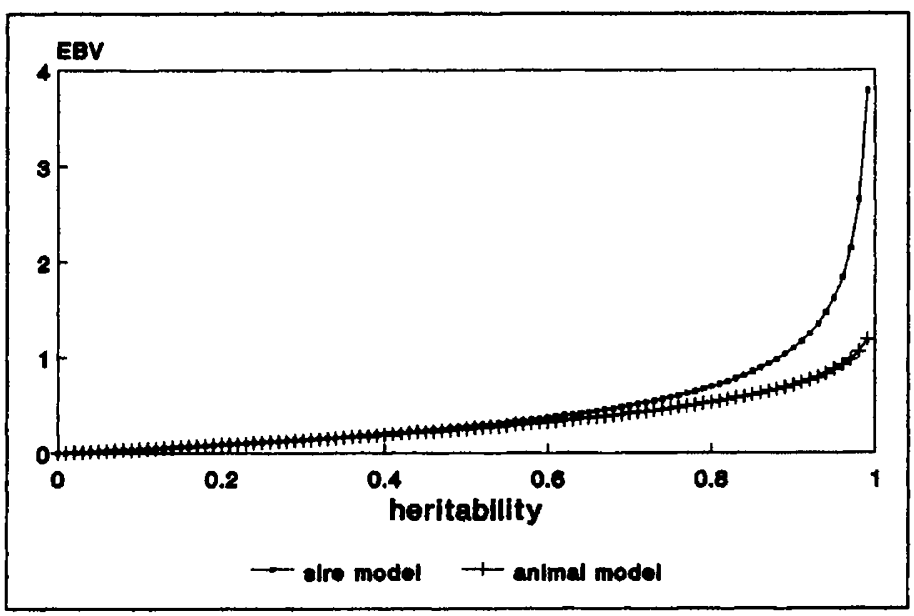

environmental sd $=1$

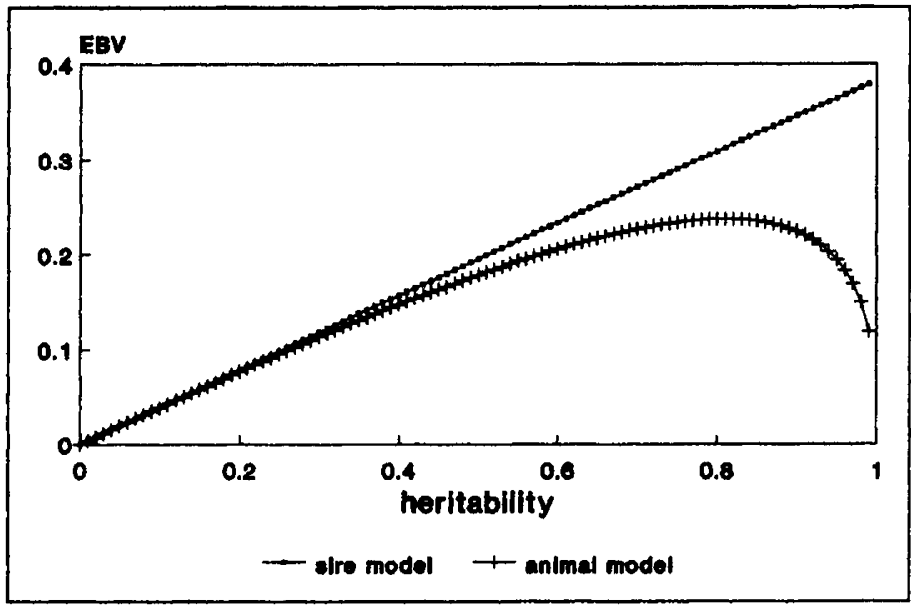

phenotypic sd = 1

Fig 1. Estimated breeding value (EBV) of sire 1 in the example data set, considering model type (sire model or animal model) and heritability.

the EBV based on a sire model shows an almost linear relationship with the heritability even if with increasing heritability the slope slightly decreases. With a higher intraclass correlation on the underlying scale as with the animal model, this phenomenon can have a drastic impact. Thus, with the animal model, the EBV 
exhibits a maximum at a heritability value of about 0.81 and decreases rapidly thereafter.

\section{Hypothetical data set by Gianola and Foulley}

Table II shows the maximum a posteriori estimates of the parameters of the hypothetical data set in table I for the 2 different model types (sire model and animal model). The estimates are expressed in units of the phenotypic standard deviation. The estimable linear function $t_{1}+H_{1}+A_{h}+S_{m}$ represents the baseline. Further, in table II the estimates of the contrasts $t_{2}-t_{1}$ (threshold 2 - threshold 1), $H_{2}-H_{1}$ (herd-year 2 - herd-year 1 ), $A_{c}-A_{h}$ (cow - heifer calving), $S_{f}-S_{m}$ (female - male calf) are shown.

Table II. Maximum a posteriori estimates of the parameters of the data set in table I, expressed in phenotypic sd, and in increasing order of calving ease, considering 2 model types (sire model and animal model) and 2 heritabilities $\left(h^{2}=0.20\right.$ and $\left.h^{2}=0.60\right)$.

\begin{tabular}{lrcrr}
\hline & \multicolumn{2}{c}{$h^{2}=0.20$} & \multicolumn{2}{c}{$h^{2}=0.60$} \\
\cline { 2 - 5 } & Sire model & Animal model & Sire model & Animal model \\
\hline$t_{1}+H_{1}+A_{h}+S_{m}$ & 0.366 & 0.348 & 0.414 & 0.331 \\
$t_{2}-t_{1}$ & 0.620 & 0.626 & 0.608 & 0.633 \\
$H_{2}-H_{1}$ & -0.289 & -0.284 & -0.400 & -0.363 \\
$A_{c}-A_{h}$ & 0.125 & 0.116 & 0.118 & 0.086 \\
$S_{f}-S_{m}$ & 0.381 & 0.369 & 0.400 & 0.341 \\
Estimated breeding values & & & & \\
$\quad$ Sire 1 & 0.159 & 0.155 & 0.360 & 0.309 \\
Sire 2 & -0.128 & -0.125 & -0.318 & -0.287 \\
$\quad$ Sire 3 & -0.239 & -0.233 & -0.558 & -0.490 \\
$\quad$ Sire 4 & 0.208 & 0.204 & 0.516 & 0.468 \\
\hline
\end{tabular}

$t_{i}$ : threshold $i, i=1,2 ; H_{j}$ : herd-year $j, j=1,2 ; A_{k}$ : age of dam $k, k=c$ (cow), $h$ (heifer); $S_{1}$ : sex of calf $l, l=m$ (male), $f$ (female).

Moreover, table II contains the EBVs of the sires, which in the case of the sire model, for reasons of comparison, were calculated as twice the estimated sire effects. If $\widehat{t}_{j}+\lambda_{i}$ represents the estimator of a particular estimable combination $i$ of the explanatory effects, then the probability of a response in the $j$ th category $\left(p_{i j}\right)$ can be estimated as $\widehat{p}_{i 1}=\Phi\left[\left(\widehat{t}_{1}+\lambda_{i}\right) / \sigma\right], \widehat{p}_{i 2}=\Phi\left[\left(\widehat{t}_{2}+\lambda_{i}\right) / \sigma\right]-\Phi\left[\left(\widehat{t}_{1}+\lambda_{i}\right) / \sigma\right]$ and $\widehat{p}_{i 3}=1-\Phi\left[\left(\widehat{t}_{2}+\lambda_{i}\right) / \sigma\right]$, where $\sigma$ is the error standard deviation (sire model) or the environmental standard deviation (animal model).

For the heritability value of 0.20 the estimators from the sire model and the animal model are quite similar. The absolute estimates from the animal model are a little smaller, with the exception of the estimated distance between the thresholds. The greatest relative difference is shown by the contrast between cow effect and heifer effect. When a heritability of 0.60 is applied, the differences between the 2 model types are more substantial. Again, the absolute estimates from the animal 
model are smaller, only the estimated distance between the thresholds is greater and the greatest relative difference is found for the contrast between cow effect and heifer effect.

\section{Different sire rankings}

Table III shows 3 data structures for which different sire rankings under the different model types (sire model and animal model) were identified.

Table III. Estimated breeding values of sires, expressed in phenotypic sd, according to model type (sire model and animal model), and sire rankings for 3 selected data structures.

\begin{tabular}{|c|c|c|c|c|}
\hline & Sire 1 & Sire 2 & Sire 3 & Sire ranking \\
\hline$n_{i j}$ & 70 & 58 & $\begin{array}{ll}0 & 2\end{array}$ & \\
\hline Sire model & 0.963 & -0.486 & -0.477 & $1-3-2$ \\
\hline Animal model & 0.824 & -0.409 & -0.416 & $1-2-3$ \\
\hline$n_{i j}$ & 80 & 85 & $\begin{array}{ll}0 & 1\end{array}$ & \\
\hline Sire model & 0.724 & -0.366 & -0.357 & $1-3-2$ \\
\hline Animal model & 0.609 & -0.299 & -0.311 & $1-2-3$ \\
\hline$n_{i j}$ & $\begin{array}{ll}6 & 1\end{array}$ & 88 & $\begin{array}{ll}0 & 1\end{array}$ & \\
\hline Sire model & 0.564 & -0.286 & -0.278 & $1-3-2$ \\
\hline Animal model & 0.484 & -0.239 & -0.245 & $1-2-3$ \\
\hline
\end{tabular}

The estimates based on the animal model are again smaller than the estimates from the sire model. The different rankings under the 2 model types are interesting. Under the sire model the EBVs of the sires 3 are clearly greater than the values of the sires 2, whereas under an animal model it is the other way around and the EBVs of the sires 3 are clearly smaller than the EBVs of the sires 2. Other data structures with different sire rankings were identified and table III shows only a few examples. It might be expected that with higher heritability values and more complex data designs this topsy-turvy situation would be even more marked.

\section{DISCUSSION}

Studies on the properties of maximum a posteriori estimators for threshold traits have been based on sire models to describe the underlying variable. Interest has mainly focused on comparisons of maximum a posteriori estimators with the estimators and predictors of linear model methods. As regards the EBVs, or more exactly sire evaluation, the comparisons have been based on empirical productmoment correlations, sire rankings, and the realized genetic response obtained from truncation selection. Meijering and Gianola (1985) showed that with a balanced random one-way classification and binary responses, the application of quasi-best linear unbiased prediction (QBLUP) and maximum a posteriori estimation (MAPE) leads to an identical ranking. With 4 categories of response and constant progeny group size, QBLUP and MAPE gave very similar sire rankings; the differences 
in the mean true breeding values of sires with either QBLUP or MAPE as selection rules were not significant. In simulation studies where the data were generated by a one-way sire model with variable progeny group size, there were noticeable differences in efficiency between QBLUP and MAPE only when the incidence was high ( $>90 \%$, binary response) and with high $(>0.20)$ heritability (Meijering and Gianola, 1985; Höschele, 1988). When applying mixed models for data simulation, MAPE was generally found to be superior in comparisons with QBLUP. This superiority depends on several factors: differences in incidence, intraclass correlation, unbalancedness of the layout, and differences in the fixed effects and the proportion of selected candidates (Meijering and Gianola, 1985; Höschele, 1988; Mayer, 1991). The relationship between the relative superiority of MAPE and the proportion of selected candidates was not found to be of a simple kind (Mayer, 1991). Somewhat in contrast to these findings in the simulation studies were the results of investigations where product-moment correlations between MAPE and QBLUP estimators were calculated (Jensen, 1986; Djemali et al, 1987; Höschele, 1988; Weller et al, 1988; Renand et al, 1990). The correlation coefficients throughout were very high. This is even more astonishing because theoretically there is no linear relationship between MAPE and QBLUP, and so even with identical sire ranking the correlation coefficient is smaller than one.

Generally, a sire model can be considered as a special case of an animal model, making very restrictive supositions. For continuously distributed traits, the use of an animal model is currently the state of the art for genetic evaluation of breeding animals. Basically, all the practical and theoretical reasons in favour of the use of an animal model instead of a sire model for traits showing a continuous distribution are also valid for threshold traits.

However, in the present paper it was clearly shown that with threshold traits, the maximum a posteriori estimators of the parameters of the underlying variable or the estimators of the breeding value are different whether a sire model or an equivalent animal model is used to describe the underlying variable. This is, of course, an unfavourable behaviour of the estimation method, but as long as the ranking of breeding animals is not affected, it would not be so problematic from a breeding point of view. As mentioned above, QBLUP and MAPE yield exactly the same ranking of sires for a one-way random model with binary responses and constant progeny group size although there is no strict linear relationship between QBLUP and MAPE. However, as was shown in this study, with maximum a posteriori estimation, even the ranking of breeding animals may depend on the model type chosen.

The result that the use of equivalent sire and animal models for the underlying variable and the use of the same estimation method lead to different estimators may be counterintuitive. Under the animal model the maximum a posteriori estimators are obtained by computing the mode of the joint posterior distribution of the 'environmental' effects and the breeding values of all the animals. Under the sire model the mode of the marginal posterior density for the sires is computed. In the analyses of the simple example data set in this study, the 'marginal' posterior density for the sires equals the joint posterior density, after integrating out the genetic effects of the progenies $\left(\nu_{1}, \nu_{2}\right): g\left(t, u_{1}, u_{2} \mid n_{i j}, h^{2}\right)=g\left(t, \nu_{3}, \nu_{4} \mid n_{i j}, h^{2}\right)=$ 
$\iint g\left(t, \nu_{1}, \nu_{2}, \nu_{3}, \nu_{4} \mid n_{i j}, h^{2}\right) \delta \nu_{1} \delta \nu_{2}$. It is well known that the posterior densities in this study are only asymptotically normal and generally do not have typical forms of distribution (see, for example, Foulley et al, 1990). Therefore the mode of the marginal posterior density function is different from the mode of the joint posterior distribution. The situation is different in the linear model case or more precisely when the likelihood follows a normal distribution. As shown by several authors (eg, Gianola et al, 1990) then, under the assumption that the variance components are known, the joint posterior density is multivariate normal, $i e$ modes and means are identical and marginal means can be derived directly from the vector of joint means.

If the maximum a posteriori estimates depend on the model type used to describe the underlying variable, as shown in this paper, there may arise the question as to which is the better estimator. In animal breeding, the best selection rule, in the sense of maximizing the expected value of the mean of the breeding values of a fixed number of individuals selected from a fixed number of candidates, is the mean of the marginal posterior distribution of the breeding values (Goffinet, 1983; Fernando and Gianola, 1986). Only because its calculation is usually thought to be unfeasible for threshold traits (Foulley et al, 1990; Knuiman and Laird, 1990), are joint posterior modes or maximum a posteriori estimators considered and regarded as an approximation to the posterior mean. For convenience and simplicity, let us assume that in the analysis of the simple example data set in this study, the value of the threshold is known to be $t=0$. The marginal posterior mean can then be calculated, not only by numerical means, but also analytically. Under the conditions that the breeding values and the environmental effects are normally, identically and independently distributed with mean 0 and variances $\boldsymbol{\sigma}_{a}^{2}$ and $\boldsymbol{\sigma}_{\varepsilon}^{2}$ respectively, and mutually independent, it follows from well-known statistical properties of the normal distribution that the marginal expected value $E\left[a \mid M_{0}\right]$ is:

$$
E\left[a \mid M_{0}\right]=\frac{\boldsymbol{\sigma}_{a}^{2}}{\boldsymbol{\sigma}_{a}^{2}+\boldsymbol{\sigma}_{\varepsilon}^{2}} \frac{\phi(0)}{\Phi(0)}\left(\boldsymbol{\sigma}_{a}^{2}+\boldsymbol{\sigma}_{\varepsilon}^{2}\right)^{1 / 2}
$$

Using the additive-genetic transmission model, where $p$ is a progeny of sire $s$ and dam $d$, and $a_{m}$ is a random variable with mean 0 and variance $\boldsymbol{\sigma}_{a}^{2} / 2$ describing the Mendelian sampling:

$$
a_{p}=1 / 2\left(a_{s}+a_{d}\right)+a_{m}
$$

the marginal breeding value of sire $1\left(u_{1}\right)$, conditional to his progeny exhibiting the realization $M_{0}\left(n_{i j}\right)$ is:

$$
\left.E\left[u_{1} \mid n_{i j}\right)\right]=E\left[a \mid M_{0}\right] / 2
$$

In table IV this marginal mean estimator is compared with the maximum $a$ posteriori estimators under the 2 different model types (sire model and animal model).

In the simple example data set of this study the maximum a posteriori estimator under the sire model is much closer to the marginal mean estimator. This is especially true for heritabilities greater than 0.25 . With heritabilities greater than 0.50 the maximum a posteriori estimator under the animal model proved to be 
Table IV. Comparison of the maximum a posteriori estimators (MAPE) considering 2 model types (sire model and animal model) and the marginal mean estimator (MME), for different heritabilities $h^{2}$ (breeding value of sire 1 in the example data set).

\begin{tabular}{lccc}
\hline $\mathrm{h}^{2}$ & $M A P E($ sire model) & MAPE (animal model) & MME \\
\hline 0.05 & 0.020 & 0.020 & 0.020 \\
0.10 & 0.040 & 0.039 & 0.040 \\
0.25 & 0.099 & 0.096 & 0.100 \\
0.50 & 0.196 & 0.179 & 0.199 \\
0.90 & 0.346 & 0.225 & 0.359 \\
0.99 & 0.379 & 0.119 & 0.396 \\
\hline
\end{tabular}

Estimators expressed in phenotypic sd.

very poor when compared with the marginal mean estimator. In a more thorough study of marginal mean estimation by Mayer (1994b), similar results were found in the analysis of the calving ease data set presented in table I. Thus, a normal density approximation may not be justified for posterior distributions when $n_{i j}$ values are small as under an animal model. Further Mayer (1994b) showed that the standard deviation of the marginal posterior density was clearly greater than the approximate standard deviation of the maximum a posteriori estimates derived from the observed or estimated Fisher information matrix.

\section{ACKNOWLEDGMENTS}

The author is grateful to the anonymous reviewers for their helpful suggestions and comments on the manuscript.

\section{REFERENCES}

Djemali M, Berger PJ, Freeman AE (1987) Ordered categorical sire evaluation for dystocia in Holsteins. J Dairy Sci 70, 2374-2384

Fernando RL, Gianola D (1986) Optimal properties of the conditional mean as a selection criterion. Theor Appl Genet 72, 822-825

Foulley JL, Gianola D, Im S (1990) Genetic evaluation for discrete polygenic traits in animal breeding. In: Advances in Statistical Methods for Genetic Improvement of Livestock (D Gianola, K Hammond, eds), Springer-Verlag, Berlin, 361-409

Gianola D, Foulley JL (1983) Sire evaluation for ordered categorical data with a threshold model. Génét Sél Évol 15, 201-224

Gianola D, Im S, Macedo FW (1990) A framework for prediction of breeding value. In: Advances in Statistical Methods for Genetic Improvement of Livestock (D Gianola, K Hammond, eds), Springer-Verlag, Berlin, 210-238

Goffinet B (1983) Selection on selected records. Génét Sél Evol 15, 91-97

Harville DA, Mee RW (1984) A mixed model procedure for analyzing ordered categorical data. Biometrics 40, 393-408

Höschele I (1988) Comparison of 'Maximum a posteriori estimation and 'Quasi-best linear unbiased prediction' with threshold characters. J Anim Breed Genet 105, 337-361 
Jensen J (1986) Sire evaluation for type traits with linear and non-linear procedures. Livest Prod Sci 15, 165-171

Knuiman MW, Laird NM (1990) Parameter estimation in variance component models for binary response data. In: Advances in Statistical Methods for Genetic Improvement of Livestock (D Gianola, K Hammond, eds), Springer-Verlag, Berlin, 177-189

Mayer M (1991) Schätzung von Zuchtwerten and Parametern bei kategorischen Schwellenmerkmalen in der Tierzucht. Habilitation thesis, Hannover School of Veterinary Sciences, Hannover

Mayer M (1994a) TMMCAT - a computer program using non-linear mixed model threshold concepts to analyse ordered categorical data. In: Proc 5th World Congr Genet Appl Livest Prod 22, 49-50

Mayer M (1994b) Numerical integration techniques for point and interval estimation of breeding values for threshold traits. In: 45th Annual Meeting of the European Association for Animal Production. Edinburgh, 5-8 September 1994

Meijering A, Gianola D (1985) Liner versus nonlinear methods of sire evaluation for categorical traits: a simulation study. Génét Sél Evol 17, 115-132

Renand G, Janss LLG, Gaillard J (1990) Sire evaluation for direct effects on dystocia by linear and threshold models. In: Proc 4rd World Congr Genet Appl Livest Prod 465-467

Stiratelli R, Laird N, Ware JH (1984) Random effects models for serial observations with binary response. Biometrics 40, 967-971

Weller JI, Misztal I, Gianola D (1988) Genetic analysis of dystocia and calf mortality in Israeli-Holsteins by threshold and linear models. J Dairy Sci 71, 2491-2501 